\title{
Hypoxia and hypoxia-mimetics attenuate the inflammatory response during murine endotoxemia
}

\author{
D Kiers ${ }^{1,2^{*}}$, R Groeneveld ${ }^{1}$, JG van der Hoeven ${ }^{1}$, GJ Scheffer ${ }^{2}$, P Pickkers ${ }^{1}$, M Kox ${ }^{1,2}$ \\ From ESICM LIVES 2015 \\ Berlin, Germany. 3-7 October 2015
}

\section{Introduction}

Hypoxia has been shown to exert immunomodulatory effects ${ }^{1}$. As oxygenation is daily practice in critical care, and the majority of critically ill patients suffer from inflammatory-related conditions, permissive hypoxia might be a novel therapeutic strategy. In addition, there are pharmacologic hypoxia-mimetics available that can replicate the hypoxia-effects without the potential drawbacks of systemic hypoxia. The hypoxic immunomodulatory effects are thought to be mediated through a group of transcription factors called hypoxia-inducible factors (HIFs) ${ }^{2}$. However, in vitro studies have demonstrated that, depending on the cell-type, these effects can be both pro- and anti-inflammatory. The net effects of hypoxia during systemic inflammation in vivo are therefore unknown.

\section{Objectives}

To determine the immunomodulatory effects of various degrees of hypoxia and hypoxia mimetics during systemic inflammation in mice.

\section{Methods}

BALB/c mice ( $\mathrm{n}=8$ per group) were placed in an air-tight cage with variable degrees of oxygen (normal (21\%), $12 \%$, $9 \%$, and $6 \%$ ), or were injected with the hypoxia-mimetic cobalt chloride $\left(\mathrm{CoCl}_{2}, 30 \mathrm{mg} / \mathrm{kg}\right.$ i.p.). After 1 hour, LPS (5 $\mathrm{mg} / \mathrm{kg}$ E. Coli endotoxin, serotype 0111:B4) or placebo $(\mathrm{NaCl} 0.9 \%)$ was administered i.p. Ninety minutes after LPS/placebo administration, rectal temperature was measured and animals were sacrificed. Blood plasma was analyzed for cytokine concentrations. Furthermore,

${ }^{1}$ Radboud University Medical Center, Department of Intensive Care Medicine, Nijmegen, Netherlands

Full list of author information is available at the end of the article
mRNA expression of interleukin (IL)-10 and the HIF-1 $\alpha$ target gene vascular endothelial growth factor (VEGF) were determined in spleen samples.

\section{Results}

As expected, LPS administration resulted in hypothermia. Hypoxia and $\mathrm{CoCl}_{2}$ also lowered body temperature, in a dose-dependent fashion (Figure 1). Hypoxia itself did not result in elevated cytokine levels in plasma. Endotoxemia resulted in increased levels of circulating pro-inflammatory cytokines Tumor Necrosis Factor (TNF)- $\alpha$, IL-6, IL-8, as well as anti-inflammatory IL-10 (Figure 2). Hypoxia and $\mathrm{CoCl}_{2}$ attenuated the endotoxin-induced proinflammatory cytokine response in a dose-dependent

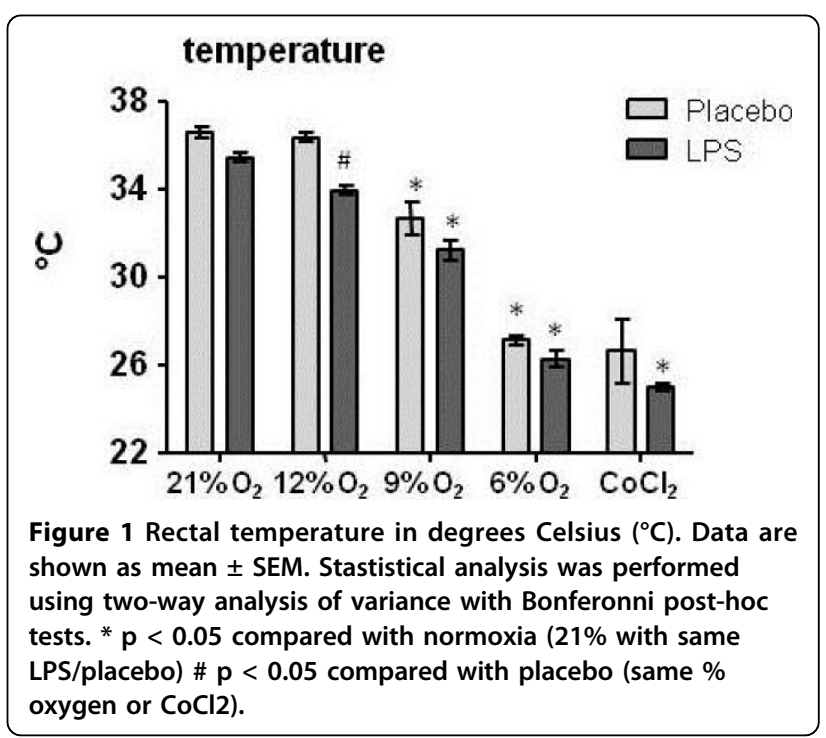



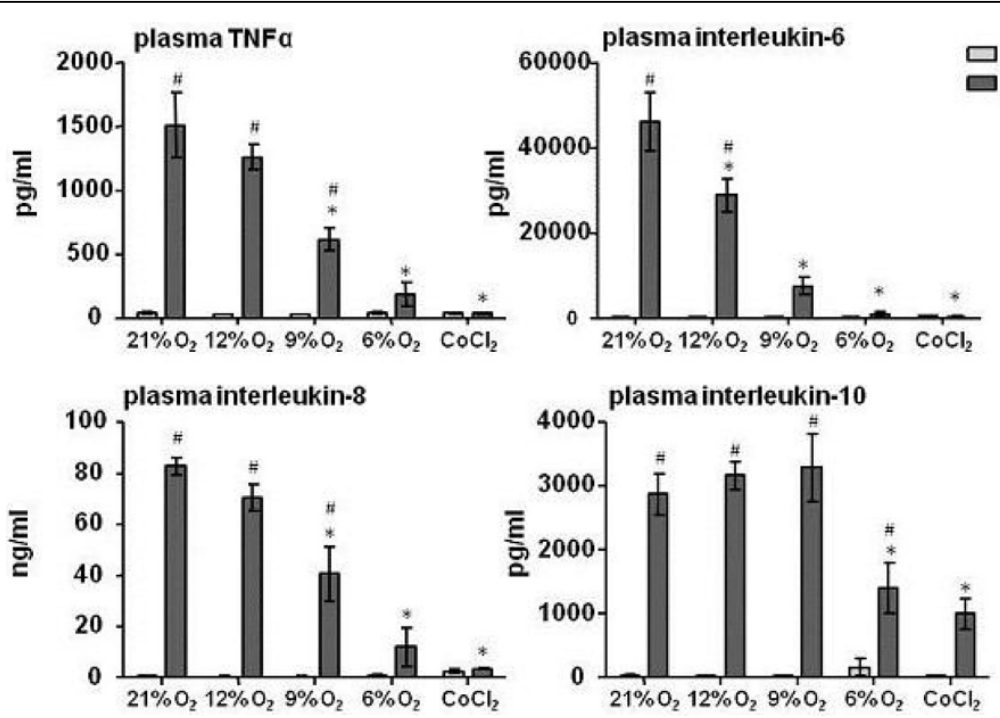

Figure 2 Plasma cytokines (Tumor Necrosis Factor (TNF) $\alpha$, Interleukin(IL)-6, IL-8 and IL-10). Data are shown as mean \pm SEM. Statistical analysis was performed using two-way analysis of variance with Bonferonni post-hoc tests. ${ }^{*} p<0.05$ compared with normoxia ( $21 \%$ with same LPS/placebo). \# $p<0.05$ compared with placebo (same $\%$ oxygen of $\mathrm{CoCl} 2$ )

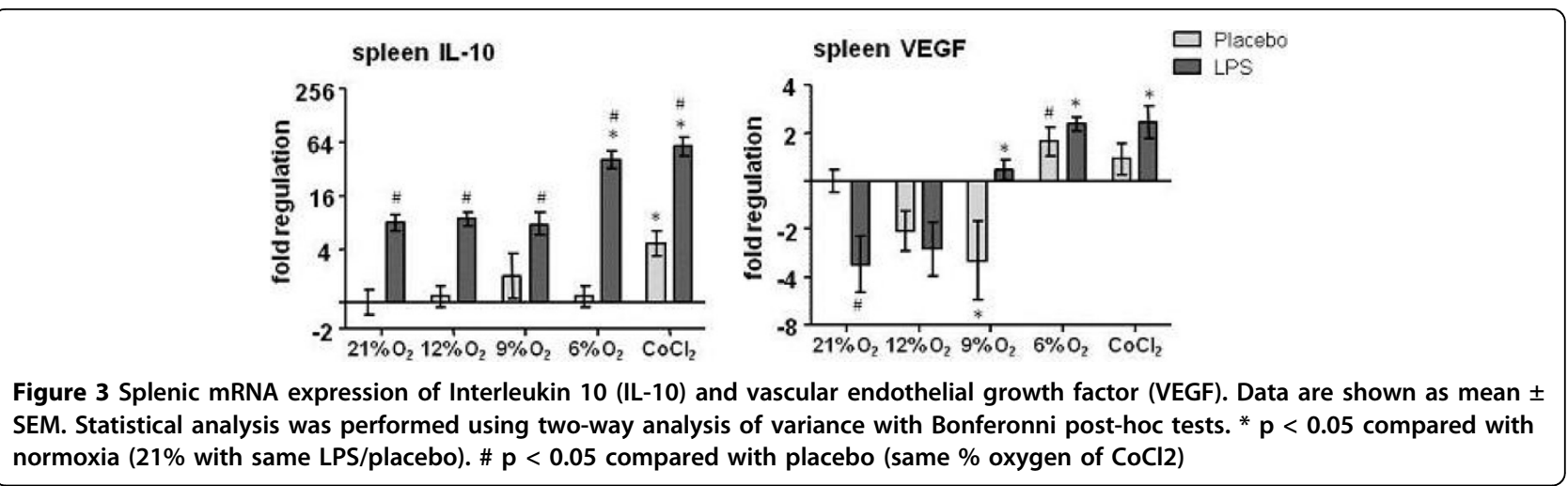

manner, while IL-10 protein levels were relatively unaffected. Furthermore, hypoxia resulted in a dosedependent upregulation of splenic VEGF and IL-10 mRNA expression (Figure 3).

\section{Conclusions}

Hypoxia results in hypothermia and attenuation of the systemic pro-inflammatory response in a dose-dependent fashion, while preserving or enhancing the anti-inflammatory response. Administration of the hypoxia-mimetic $\mathrm{CoCl}_{2}$ results in a similar immunological phenotype. Our results suggest that permissive hypoxia is a novel nonpharmacological anti-inflammatory therapeutic strategy.
Published: 1 October 2015

\section{References}

1. Eltzschig HK, Carmeliet P: Hypoxia and Inflammation. N Engl J Med 2011, 364:656-665.

2. Palazon A, Goldrath AW, Nizet V, Johnson RS: HIF Transcription Factors, Inflammation, and Immunity. Immunity 2014, 41(4):518-528.

3. Eltzschig HK, Sitkovsky MV, Robson SC: Purinergic signaling during inflammation. N Engl J Med 2012, 367:2322-2333.

doi:10.1186/2197-425X-3-S1-A421

Cite this article as: Kiers et al:: Hypoxia and hypoxia-mimetics attenuate the inflammatory response during murine endotoxemia. Intensive Care Medicine Experimental 2015 3(Suppl 1):A421. 